\title{
Designing Collaborative Interactive Spaces
}

\author{
Hans-Christian Jetter, \\ Florian Geyer, Harald Reiterer \\ Human-Computer Interaction Group, \\ University of Konstanz, Germany \\ \{lastname\}@inf.uni-konstanz.de
}

\author{
Raimund Dachselt \\ Multimedia Technology, Dresden \\ University of Technology, Germany \\ dachselt@acm.org
}

\author{
Gerhard Fischer \\ Center for Lifelong Learning and \\ Design, University of Colorado, USA \\ gerhard@colorado.edu
}

\author{
Thomas Herrmann \\ Chair for Information- and \\ Technology Management \\ Ruhr University Bochum, Germany \\ herrmann@iaw.ruhr-uni- \\ bochum.de
}

\begin{abstract}
Interactive spaces are ubiquitous computing environments for computer-supported collaboration that exploit and enhance the existing cognitive, physical and social skills of users or groups of users. The workshop aims at documenting and advancing the current state-of-the-art of co-located collaboration in interactive spaces and identifying research challenges and formulating a research agenda by inviting high-quality position and research papers from HCI, Information Visualization, CSCW and CSCL.
\end{abstract}

\section{Categories and Subject Descriptors}

H.5.3 Group and Organization Interfaces: collaborative computing, computer supported cooperative work

\section{General Terms}

Design, Experimentation, Theory, Verification.

\section{Keywords}

Interactive Spaces, Collaboration, Ubiquitous Computing, Interactive Surfaces, Tangible Interfaces.

\section{INTRODUCTION}

In the recent years, we have witnessed a dramatic evolution of user interfaces (UIs) from command-based languages and graphical UIs to natural and reality-based interaction [7]. Backed by an embodied view of cognition and interaction [2], influential visions like ubiquitous computing [15] and augmented reality [16] have contributed to a more holistic understanding of humancomputer interaction that goes far beyond the traditional WIMP (Windows, Icons, Menu, Pointer) paradigm of an isolated single user in front of a mouse- and keyboard-operated personal computer in an office environment. Instead, the embodied interaction with ubiquitous computing has become "tangible" and "social" [2] and is integrated into our physical and social environment using wireless networks and post-WIMP hardware with computer vision and touch or motion tracking. Multi-touch enabled tabletops, digital whiteboards and mobile devices such as the Apple iPad or iPhone complement the desktop PC, so that computation is now deeply woven into the fabric of our entire private and professional life.

Embodied views of cognition and interaction also teach us that our interaction with our physical and social environment, e.g., with physical objects or other individuals, is defining the ways in which we think and reason about the world. This is not only true for the way in which we think and reason about the real nondigital world, but it also inevitably defines the ways in which we are able to conceptualize, understand, use and adopt digital technology and its virtual functionality. It is therefore important to always keep our non-digital bodily and social experiences, skills \& practices in mind when designing post-WIMP UIs.

As a consequence, interaction designers strive to make interactive systems more "natural", for example with haptic and gestural interfaces that rely on our real-world motor skills and gestural communication. Microsoft's Kinect gaming controller facilitates the creation of commercial multi-modal user interfaces [12] that use body movement, gestures and voice as input. Furthermore, tangible user interfaces (TUIs) [6] are becoming increasingly important for products such as Sifteo Cubes or Microsoft Surface tabletops that do not only influence HCI researchers, but also the work practice of end-users. Using hybrids of TUIs and multitouch user interfaces on tabletops can not only help to exploit our natural motor skills, but can also afford natural face-to-face collaboration with flexible working styles and increased group awareness [9]. Also, techniques of information visualization can help to better exploit the highly developed human visual perception and pattern recognition to achieve a more fluid and efficient interaction with large amounts of data [3]. In the recent years, this wide range of different designs, techniques, and technologies became available to the designers of collaborative interactive spaces at a breathtaking speed. 


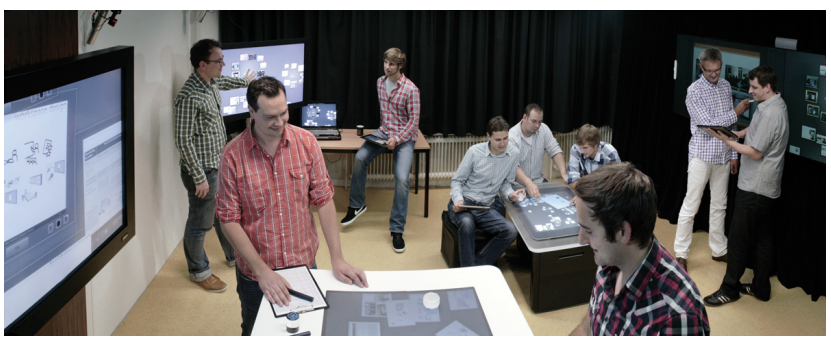

Figure 1. A multi-user, multi-display and multi-device interactive space at the University of Konstanz [13].

Interactive spaces are ubiquitous computing environments for computer-supported collaboration that exploit and enhance the existing cognitive, physical and social skills of users or groups of users. In contrast to traditional WIMP computing, the underlying human-computer interaction is integrated seamlessly into established work practices and into the work environment to achieve a natural, unobtrusive support for activities such as presentation, brainstorming, sense-making or data analysis. The successful design of such interactive spaces poses a wide range of challenges that are not only concerned with technological issues but also with still unanswered research questions of HCI and unsolved problems of interaction design.

While the original vision of 'interactive spaces' can be traced back to seminal work such as Streitz et al.'s i-Land [14] or the Stanford iRoom [10], many findings about the theory, design and practice of interactive spaces have to be re-evaluated today. In particular, due to the recent advances in commercially available hardware and software technology (novel tabletops such as Microsoft Surface 2.0, light high-performance tablets and tablet PCs based on Android or Windows, pico projectors \& depth cameras for gestural and "touch anywhere" interaction, operating systems bridging mobile and stationary devices, e.g. Microsoft Windows 8 ), this field moves quickly and formerly impossible designs and visions can now be put into practice. This new generation of interactive spaces with natural user interfaces is of great relevance in knowledge-intensive and collaborative application domains such as e-Creativity [4,5], e-Science [11] and e-Learning [1].

\section{GOALS OF THE WORKSHOP}

The workshop aims at documenting and advancing the current state-of-the-art of co-located collaboration in interactive spaces and identifying research challenges and formulating a research agenda by inviting high-quality position and research papers from HCI, Information Visualization, CSCW and CSCL. This multidisciplinary perspective is intended to provide a more comprehensive overview beyond the typical boundaries of the individual fields.

The workshop's themes include but are not limited to:

- Understanding users' natural collaboration in rooms when using interactive spaces or non-digital physical artifacts.

- Conceptual frameworks for natural user collaboration, learning and interaction.

- Novel designs of interaction techniques, visualizations, smart furniture or devices for natural collaboration in interactive spaces.
- Enabling technologies for natural interaction in interactive spaces ranging from software frameworks to computer vision and new sensor technology.

- New observation and evaluation approaches for interactive spaces (e.g. longitudinal evaluation).

- Best-practice examples and lessons learned from different application domains based on the experiences of academics and industry.

\section{WORKSHOP ORGANIZATION}

The workshop is organized into long talks $(20$ minute presentations, 5 minute discussions) and short talks (10 minute presentations, 5 minute discussions) for each peer-reviewed submission, followed by a 45-60 minute open discussion for conclusions at the end of the workshop. The aim of the final discussion is to identify a future research agenda.

\subsection{Organizers}

The workshop organizers are all active in the field of interactive spaces and environments. In their research, they designed, implemented, and evaluated several interactive spaces for supporting collaborative work within different domains and for different user populations, e.g., novices vs. experts. Thereby, they have put their designs to the test in a broad range of domains such as creative design, libraries, museums, scientific research, and control rooms $[1,4,5,8,9,11,13]$.

\subsection{Program Committee}

We employed a single-blind review process for selecting papers from the submissions. We thank the members of the program committee:

Raimund Dachselt, University of Magdeburg, Germany.

Stefan Dierdorf, University of Konstanz, Germany.

Gerhard Fischer, University of Colorado at Boulder, USA.

Florian Geyer, University of Konstanz, Germany.

Rainer Groh, Media Design, Technische Universität Dresden, Germany.

Michael Haller, Upper Austria University of Applied Sciences, Austria.

Thomas Herrmann, Ruhr University Bochum, Germany.

Hans-Christian Jetter, University of Konstanz, Germany

Roman Rädle, University of Konstanz, Germany.

Harald Reiterer, University of Konstanz, Germany.

Tobias Schwarz, University of Konstanz, Germany.

Sophie Stellmach, University of Magdeburg, Germany.

Peter Thomas, Manifesto Group, London, Melbourne \& New York.

\subsection{Dissemination}

All accepted submissions are published on the workshop website ${ }^{1}$. Extended versions of selected submissions will be published in a forthcoming special issue of the Springer journal 'Personal and Ubiquitous Computing, 2 .

\footnotetext{
1 http://hci.uni-konstanz.de/dcis/

${ }^{2}$ http://www.springer.com/computer/hci/journal/779
} 


\subsection{Workshop Participants}

Our participants are researchers from academia (professors, postdocs, PhD students) and researchers from research labs. From 24 submissions we were able to accept only 12 papers for presentation (50\% acceptance rate). The accepted workshop contributions are from a range of international organizations and universities:

- Centre for Interaction Design, Edinburgh Napier University, UK

- Dresden University of Technology, Germany

- Eindhoven University of Technology, Department of Industrial Design, The Netherlands

- ENAC/ DGAC DSNA DTI R\&D / IRIT, Toulouse, France

- Heudiasyc Laboratory / COSTECH Laboratory, University of Technology of Compiègne, France

- Information and Technology Management, RuhrUniversity of Bochum, Germany

- Institute for Energy Technology, Halden, Norway

- Instrata Ltd. / Microsoft Research, Cambridge, UK

- Media Interaction Lab, Hagenberg, Austria / University of Calgary, Canada

- University of Konstanz, Germany

- University of Magdeburg, Germany

\section{ACKNOWLEDGMENTS}

We thank Microsoft Research Limited, Cambridge, UK for supporting this workshop.

\section{REFERENCES}

[1] Arias, E., Eden, H., Fischer, G., Gorman, A. and Scharff, E. 2000. Transcending the individual human mind - creating shared understanding through collaborative design. $A C M$ Trans. Comput.-Hum. Interact. 7, 1 (March 2000), 84-113.

[2] Dourish, P. 2001. Where The Action Is: The Foundations of Embodied Interaction. MIT Press. Cambridge, MA, USA.

[3] Elmqvist, N., Moere, A., Jetter, H-C., Cernea, D., Reiterer, H., Jankun-Kelly, T. J. 2011. Fluid Interaction for Information Visualization. in: Chaomei Chen, Ben Shneiderman, Sage, Information Visualization, p. 327-340.

[4] Geyer, F., Pfeil, U., Höchtl, A., Budzinski, J. and Reiterer, H. 2011. Designing reality-based interfaces for creative group work. In Proceedings of the 8th ACM conference on Creativity and cognition (C\&C '11). ACM, New York, NY, USA, 165-174.
[5] Herrmann, T. and Nolte, A. 2010. The integration of collaborative process modeling and electronic brainstorming in co-located meetings. In Proceedings of the $16^{\text {th }}$ international conference on Collaboration and technology (CRIWG'10), Springer-Verlag, 145-160.

[6] Ishii, H., Ullmer, B. 1997. Tangible Bis: Towards Seamless Interfaces between People, Bits and Atoms. In Proceedings of the SIGCHI conference on Human factors in computing systems. CHI 2007. ACM Press, p. 234-241.

[7] Jacob, R. J., Girouard, A., Hirshfield, L. M., Horn, M. S., Shaer, O., Solovey, E. T., and Zigelbaum, J. 2008 Realitybased interaction: a framework for post-WIMP interfaces. In Proceeding of the SIGCHI Conference on Human Factors in Computing Systems. CHI '08. ACM, p. 201-210.

[8] Jetter, H.-C., Gerken, J., Zöllner, M., Reiterer, H. and MilicFrayling, N. 2011. Materializing the query with facetstreams: a hybrid surface for collaborative search on tabletops. In Proceedings of the 2011 annual conference on Human factors in computing systems (CHI '11). ACM, New York, NY, USA, 3013-3022.

[9] Jetter, H. C., Gerken, J., Zöllner, M. and Reiterer, H. 2010. Model-based design implementation of interactive spaces for information interaction, Proc. Human-Centred Software Engineering (HCSE '10), Springer, 22-37.

[10] Johanson, Brad, Fox, Armando, and Winograd, T. 2002, The Interactive Workspaces project: experiences with ubiquitous computing rooms, IEEE Pervasive Computing, 1, 67-74.

[11] Milic-Frayling, N., Oleksik, G., Jones, R., Jetter, H.-C., Gerken, J., Reiterer, H. and Baumberg, J. 2010. DeskPiles, http://research.microsoft.com/en-us/projects/deskpiles/, accessed Aug 23, 2011.

[12] Oviatt, S. 2008. Multimodal Interfaces. In Sears, A., Jacko J. (Eds.) The Human-Computer Interaction Handbook (2nd Edition), Lawrence Erlbaum Associates, New York, 2008, p. 413-432.

[13] Reiterer, H. 2011. Human-computer interaction group: University of Konstanz, Germany. interactions 18, 6 (November 2011), 82-85.

[14] Streitz, N.A., Geißler, J., Holmer, T., Konomi, S., MüllerTomfelde, C., Reischl, W., Rexroth, P., Seitz, P. and Steinmetz, R. 1999. i-LAND: an interactive landscape for creativity and innovation. In Proceedings of the SIGCHI conference on Human factors in computing systems: the CHI is the limit (CHI '99). ACM, New York, NY, USA, 120-127.

[15] Weiser, M. 1991. The Computer for the Twenty-First Century. In Scientific American. September 1991, p. 94-100.

[16] Wellner, P. 1993 Interacting with paper on the DigitalDesk. Communications of the ACM, Vol. 36, Iss. 7, p. 87-96. 